\title{
Ontology for Collapse Theories
}

\author{
Wayne C. Myrvold \\ Department of Philosophy \\ The University of Western Ontario \\ Forthcoming in Shan Gao, ed., Collapse of the Wave Function, \\ Cambridge University Press.
}

\begin{abstract}
In this chapter, I will discuss what it takes for a dynamical collapse theory to provide a reasonable description of the actual world. I will start with discussions of what is required, in general, of the ontology of a physical theory, and then apply it to the quantum case. One issue of interest is whether a collapse theory can be a quantum state monist theory, adding nothing to the quantum state and changing only its dynamics. Although this was one of the motivations for advancing such theories, its viability has been questioned, and it has been argued that, in order to provide an account of the world, a collapse theory must supplement the quantum state with additional ontology, making such theories more like hidden-variables theories than would first appear. I will make a case for quantum state monism as an adequate ontology, and, indeed, the only sensible ontology for collapse theories. This will involve taking dynamical variables to possess, not sharp values, as in classical physics, but distributions of values.
\end{abstract}

\section{Introduction}

The issue of how to interpret dynamical collapse theories is one that has been much discussed in the literature. As originally construed, such theories were taken to be ones according to which the wave function is everything (it would be better to say: physical reality is exhausted by whatever it is in the physical world that the wave function represents). The viability of this has been questioned, and it has been argued that dynamical collapse theories, in order to afford a description of a world anything like the one we live in, must be supplemented by additional ontology, making such theories more akin to the de Broglie-Bohm pilot wave theory than they might originally have seemed. ${ }^{1}$ If one accepts such an argument, then one is left with a choice to make of primitive ontology, resulting in distinct, but empirically equivalent, theories, depending on choice of ontology.

This chapter will not attempt to survey these discussions. Rather, I will try to make a case that the appropriate way to think of such theories -indeed, I will argue, the only sensible way - is still quantum state monism. On a quantum state monist

\footnotetext{
${ }^{1}$ Allori et al. (2008) is the locus classicus.
} 
approach there is nothing over and above the quantum state. There must, however, be physical objects such as tables and chairs and laboratory equipment. If the theory is to describe a world in which there are such things, a quantum state monist must adopt the proposal, made early on in these discussions, of revising the way we attribute physical properties to systems, from the strict eigenstate-eigenvalue link to a slightly relaxed criterion on which we can talk of a system possessing a property if the state is close enough to an eigenstate of the operator corresponding to that property. These possessed properties do not, however, exhaust the ontology; a full account of the world, according to these theories, is one on which dynamical quantities almost never have precise values, but rather, have distributions of values, an account that has been developed and advocated by Philip Pearle (2009).

We will begin with some general considerations about what it takes for a physical theory to give an account of the world. I will first argue for the need for what Bell called "local beables," and then proceed with an analysis, which will draw heavily on Newton's own thoughts on the matter, of what is required in order for a theory to represent a world of physical objects located in space.

Many of the discussions about these matters have invoked conformity to common sense or to intuition as a means to judge the acceptability of proposals concerning ontology. This, it seems to me, is an inadequate basis for making such judgments. We have no reason to expect either common sense or intuition to be reliable guides to what the world is like, or to what the world could be like. Our common sense and intuitions are likely to be shaped by contact with aspects of the world in which quantum phenomena do not reveal themselves and things behave quasi-classically. Further, it should be uncontroversial that quantum mechanics is counterintuitive in some aspect or another. And even when it comes to classical physics, our intuitions can lead us astray. Some readers will, I expect, take the Newton-inspired account of physical objects offered in section 3 , below, to violate some of their intuitions about what things are. This should not be taken as an objection; as always, when we find ourselves in the grip of an intuition, we should ask of it whether it can survive scrutiny and be turned into a considered judgment. Any intuitions one might have that are at odds with the account given, I claim, fail to survive such scrutiny.

For an argument for quantum state monism, along different lines but to a conclusion that I take to be compatible with, and complementary to, the one advanced in this chapter, see Peter J. Lewis' contribution to this volume (Lewis, 2017). For a contrary view, see Roderich Tumulka's contribution (Tumulka, 2017).

\section{The need for things to be met with in space}

What is required for a sensible ontology for a physical theory? We must certainly not presuppose that the world must conform to our prejudices about the way things are. If there is one thing that we have learned from the history of science, it is that science can teach us that the world differs in some fairly drastic ways from what we might have otherwise thought.

We are open to learning that the world is radically different from what everyday experience or the science of ages past might suggest. Does this entail an "anything goes" attitude towards the ontology of physical theory? Not quite. The conclusions of science, radical as they may be, gain their credibility, and their right to be taken 
seriously, by virtue of being supported by empirical evidence. The process of gathering such evidence involves manipulation of experimental and observational apparatus, and recording of the results of experiments and observations, which are then used as input for theory construction and theory testing (and, of course, this is a dialectical process, as theory informs and guides our experimentation and observation). If, via this process, one arrived at a theory with no room for any such things as experimental apparatus in its ontology, one would find oneself in an awkward position, as the theory would undermine its own empirical base. This would be peculiar situation. Though there is no logical contradiction involved in the supposition that everything that we have taken to be an experimental result is in fact an illusion, we should bear in mind that what we are pursuing is not merely a logically consistent account of the world, but one that we have some reason to believe is at least approximately correct in its broad outlines. This cannot be if the theory is empirically self-undermining. To say that a theory is empirically self-undermining does not entail that it is false, but it does entail that we do not have good reason to believe in its truth.

Our empirical evidence is based on experience. As Tim Maudlin (2007) has emphasized, it does not follow that an adequate physical theory entail facts about experiences of conscious beings. Physics proceeds while the mind-body problem remains a vexed question. Our evidence is based on manipulations and observations of experimental apparatus, and, if we are able to represent this apparatus within the theory, at least in a schematic way, so that we are in a position to compare how the apparatus is observed to behave with what the theory would lead one to expect of it, then we are in a position to test the theory empirically, and gain evidence for or against the theory.

This is nothing new. Consider, for example, the case of Newtonian gravitational theory, applied to the solar system. The evidence base is observations of planetary positions from various observatories. The theoretical treatment consists of positing a mass distribution - which consists, for example, of representations of the planets and the sun by means of points with which are associated appropriate masses - and studying the evolution of this mass distribution under gravitational interactions. Once this is done, there is still the matter of connecting the theory with observations. There is a superb discussion of this by Howard Stein, in a paper entitled, "Some Reflections on the Structure of Our Knowledge in Physics."

Let me underscore the point that there can be no thought of deducing observations within that framework. To do so in the strict sense, one would need to have a physical theory of the actual observer, and to incorporate it into the Newtonian framework. I certainly do not want to say that there is a reason "in principle" why such a thing can never be done, for any possible (future) physical framework; but everyone knows that Newton could not do it, and that we - in the best versions of our own physics - cannot do it. Even waiving the theory of the observer, it is clear that all astronomical observations are intermediated by light; therefore, to deduce anything like observations, one would have to include the theory of light within the framework (Stein, 1994, p. 649).

What we do, instead, is represent the observer (or, more accurately, the observatory) schematically within the theory.

One represents the observer within the spatio-temporal framework by a worldline (or a system of world-lines). Putting in-for the gravitational 
theory of the solar system - the world-lines of the planets and satellites, as calculated from suitable initial data, one can then determine at each instant all the relevant angles between lines drawn from the observer to the bodies of the system (including, if the theory is properly handled, with the earth represented as an extended body and its rotation treated systematically, lines from the observer to terrestrial landmarks). As a first approximation, such lines are treated as lines of sight. With more sophistication on the observational side, the results are turned over to the experts in observational astronomy, who will take such account as they are able to of atmospheric refraction, of aberration of starlight, and so on (p. 650).

A few comments about this. First of all, there can be no question of trying to ascertain, from the mathematical structure of the theory alone, what the components of the mathematical structure are supposed to represent. One knows, from the beginning, which world-line is meant to be the world-line of the Sun, which of Jupiter, of the Earth, etc.. The formalism is from the outset a physically interpreted formalism. Some philosophers have imagined a set of explicit "correspondence rules" providing the link between the formalism employed in physical theory and the physical systems to be represented. There is something right about this, but also something misleading. Physicists' treatments of physical systems typically include verbal descriptions of what it is that the variables introduced into the analysis are meant to represent. But, as Stein himself rightly emphasizes, the connection of the theoretical apparatus to observational practice is never wholly explicit; it is something learned in practice, and, for the case of laboratory experiments, in the lab, via actual experience with the relevant instruments.

Second, Newtonian gravitational theory is a theory only of a limited aspect of physical phenomena. As Stein emphasizes, it does not treat of the behaviour of light or of the interactions of ponderable matter with light, and, as such, does not treat of the key attribute of the Sun and the planets that permits us to gain information about where they are. But it is an intelligible project to extend the theory so as to include these aspects, and to embed Newtonian gravitational theory within a theory that also treats of light and its interactions with matter. Moreover, any theory with any pretensions of being in principle a comprehensive account of the physical world would have to include this extension.

At the close of the paper, Stein adds some remarks about quantum mechanics. "In this theory, we just do not know how to 'schematize' the observer and the observation" (p. 653). It might be better to say: we do not know how to schematize experimental apparatus and experimental results. That this is not a straightforward matter within the usual framework of the theory is precisely what gives rise to the so-called measurement problem. More on this in $\S 4$, below.

\section{What is it to be a material body?}

\subsection{The Newtonian conception of bodies}

It must, therefore, be possible to represent, at least schematically, within a theory that has the potential to be a comprehensive one, the sorts of things that our experimental devices are. Let's pause for a moment and ask: what does it take to be such a thing? 
On this matter, there is an interesting discussion, at the very birth of modern physics, by Isaac Newton, in a manuscript entitled "De gravitatione et aquipondio fluidorum," unpublished until 1962. In this manuscript Newton attacks, among other things, the Cartesian doctrine that the essential attribute of body is extension. Newton distinguishes body from extension, and, indeed, accepts that there are extensions - that is, extended regions of space - that are not the locations of bodies. What, then, we may ask Newton, is the difference between extended regions that are the locations of bodies, and those that are not?

To explain his answer to this question, Newton presents a sort of creation myth.

it must be agreed that God, by the sole action of thinking and willing, can prevent a body from penetrating any space defined by certain limits.

If he should exercise this power, and cause some space projecting about the Earth, like a mountain or any other body, to be impervious to bodies and thus stop or reflect light and all impinging things, it seems impossible that we should not consider this space to be truly body from the evidence of our senses (which constitute our sole judge on this matter); for it will be tangible on account of its impenetrability, and visible, opaque and coloured on account of the reflection of light, and it will resonate when struck because the adjacent air will be moved by the blow.

Thus we may imagine that there are empty spaces scattered through the world, one of which, defined by certain limits, happens by divine power to be impervious to bodies, and ex hypothesi it is manifest that this would resist the motions of bodies and perhaps reflect them, and assume all the properties of a corporeal particle, except that it will be motionless. If we may further imagine that that impenetrability is not always maintained in the same part of space but can be transferred hither and thither according to certain laws, yet so that the amount and shape of that impenetrable space are not changed, there will be no property of body which this does not possess. It would have shape, be tangible and mobile, and be capable of reflecting and being reflected, and no less constitute a part of the structure of things than any other corpuscle, and I do not see that it would not equally operate on our minds and in turn be operated upon ...

... In the same way if several spaces of this kind should be impervious to bodies and to each other, they would all sustain the vicissitudes of corpuscles and exhibit the same phænomena. And so if all this world were constituted of this kind of beings, it would hardly seem to be any different in character. And hence these beings will be either bodies or like bodies. If they are bodies, then bodies can be defined to be determinate quantities of extension which the omnipresent God affects with certain conditions: these are (1) that they be mobile (and therefore I have not declared them to be numerical parts of space, which are strictly immobile, but only definite quantities which may be transferred from space to space). (2) That two such be unable in any part to coincide, or that they be impenetrable and so when by their motions they meet they obstruct one another and are reflected in accordance with certain laws. (3) That they be able to excite various perceptions of the senses and the fancy in created minds, and in turn to be moved by the latter .... (Newton, 1962, pp. 139-140). 
Some readers, familiar with the all-too-common attribution of spacetime substantivalism to Newton, will be tempted to gloss Newton's ontology as: the basic ontology is space, with the properties that are characteristic of bodies, impenetrability, opacity, and the like, being attributes of some regions of space. As if anticipating this reading, Newton takes pains to make it clear that he rejects it.

Perhaps now it may be expected that I should define extension as substance or accident or else nothing at all. But by no means, for it has its own manner of existence which fits neither substances nor accidents. It is not substance...

... although philosophers do not define substance as an entity that can act upon things, yet all tacitly understand this of substances, as follows from the fact that they would readily allow extension to be substance in the manner of body if only it were capable of motion and of sharing in the action of body. And on the contrary they would hardly allow that body is substance if it could not move nor excite in the mind any sensation or perception whatever (Newton, 1962, pp. 131-132).

Space itself is not a substance. Where the requisite dynamical conditions hold, there is a material body, and Newton explicitly denies that those conditions must inhere in some substantival substrate, itself devoid of properties, which Newton rejects as unintelligible.

for the existence of these beings it is not necessary that we suppose some unintelligible substance to exist in which as subject there may be an inherent substantial form ....

Between extension and its impressed form there is almost the same analogy that the Aristotelians posit between the materia prima and substantial forms, namely when they say that the same matter is capable of assuming all forms, and borrows the denomination of numerical body from its form. ... They differ, however, in that extension (since it is what and how constituted and how much) has more reality than materia prima, and also in that it can be understood, in the same way as the form that I assigned to bodies (Newton, 1962, pp. 140-141).

A few comments about this "creation myth" of Newton's are in order. First, however central the agency of God was to Newton's thought about the physical world, reference to divine decree is not essential to the account of body that he presents. What matters is that it be a matter of physical law that the requisite conditions hold in the relevant regions, whether or not one takes those laws to issue from divine decree. Second, though Newton talks as if there is an absolute distinction between rest and motion, nothing in the account requires this, which is equally at home in Galilean or Minkowski or some other relativistic spacetime.

Third, the various dynamical properties, such as opacity, reflectivity and impenetrability, can be separated. For example, a body need not be impermeable, or may be permeable to some sorts of things and not others, or permeable to various degrees to various sorts of things. Though Newton took it for granted Jupiter and Saturn are impenetrable, and seemed to assume that his readers would, too, ${ }^{2}$ we now know that

\footnotetext{
${ }^{2}$ See the remarks to the Third Rule for the Study of Natural Philosophy in the Principia: "We find those
} 
they are giant balls of gas. They nonetheless share with other extended bodies the properties of reflectivity and mobility.

Fourth, as illustrated by the cases of Jupiter and Saturn, the region occupied by a body need not have sharp boundaries. Sharp as the visual boundary of Jupiter nay seem to be through your favourite telescope, the upper atmosphere does not a precise upper boundary, and any precise delimitation of it would have to involve an element of convention. One might, perhaps, imagine that the atmosphere is composed of smaller parts, atoms or molecules, which themselves have precise regions that they occupy, and that any ambiguity in the boundary of Jupiter has to do only with which of these well-located particles to include as its parts. But there is no necessity for bodies to be composed of parts like this. All of the dynamical properties that make a body a body are a matter of degree, and it would not detract at all from their status as bodies if the intensity of these properties did not go abruptly to zero outside a precisely defined region but, rather, dropped off sharply, quickly becoming negligible outside a region that is only specifiable within some tolerable limits of fuzziness.

Fifth, though Newton expresses some doubt about whether the bodies that make up our world are the kind of being he describes, he rightly says that, if this world were constituted of this kind of being, it could hardly be said to be any different in character. Mobile quantities of extension endowed with the right sorts of dynamical properties would be bodies, whatever else might be true of them, and, without the right sort of dynamical properties, nothing could be said to be a body or to have a location in space.

With these qualifications, there seems to something deeply right about Newton's account of body. To be a body is to have a certain sort of place in a network of dynamical relations. A dynamically inert substrate for dynamical properties is neither needed nor intelligible. Moreover, these considerations are independent of the details of physical theory, and may justifiably be taken as a constraint on any physical theory that has any pretense to include in its ontology things to be met with in space.

One consequence of the Newtonian view of a physical body is worth mentioning. One might be tempted to consider, as a skeptical hypothesis, a supposition that there is, in fact, no table in front of me, merely a region of empty space that, by God's decree, looks and feels and in all ways like a table. Our Newton-inspired conception of physical body declares this to be nonsensical. There is nothing more to being a table than to act in every way like a table.

\subsection{Is talk of "primitive ontology" helpful?}

In an influential and in many ways insightful paper, Allori et al. (2008) argue that the ontology of any physical theory can be partitioned into primitive ontology, which is meant to be what the theory is primarily about, and non-primitive ontology, whose role it is to provide dynamics for the primitive ontology.

I understand the motivation for talk of this sort in the context of Bohmian mechanics. This theory is primarily about the motion of the Bohmian corpuscles, and the role of the wave function is to guide the motion of these corpuscles. It is less clear

bodies that we handle to be impenetrable, and hence we conclude that impenetrability is a property of all bodies universally." (Newton, 1999, p. 795). This is explicitly extended to the heavenly bodies in the last paragraph of the remarks on Rule 3. 
to me that all physical theories fall naturally into this mode. And there seem to me to be some risks associated with this way of thinking. One risk is to fall into thinking that the local beables that make up ordinary objects must be part of the fundamental ontology of the theory. This is not a condition placed on the ontology in Allori et al. (2008), but it is a natural extension of this way of thinking, and it is taken as a requirement in Allori (2013). Another risk is regarding the non-primitive ontology as unreal. Again, this is not a step by taken in Allori et al. (2008), but it is taken in Allori et al. (2014, p. 332).

The most serious danger, though, it seems to me, is the danger of sliding into thinking that there is a two-step process. First, one posits an ontology, with no dynamical assumptions, that is, no assumptions about how it behaves, and then one posits a dynamics for it. This suggests that it could make sense to speaking of something being present that is dynamically inert. This, again, is not a pitfall that Allori et al. (2008) fall into, though they come close when they say, "The wave function in each of these theories, which has the role of generating the dynamics for the PO [primitive ontology], has a nomological character utterly absent in the PO" (Allori et al., 2008, p. 363). This is, however, a step taken by Esfeld (2014) and by Esfeld et al. (2017), who posit "primitive stuff, materia prima, having no physical properties at all" (p. 135), matter points possessing only metrical relations to each other.

The problem with this is that, until we have said something about how the purported ontology acts, we have haven't yet given sense to the claim that it is there at all. What it is for an object to occupy a region of space, or, indeed, to have any sort of spatial relations to anything, is for it to do something there - exclude other objects, or reflect light, or something of the sort.

There is a potentially misleading analogy with classical planetary physics. Eudoxus, Ptolemy, Copernicus, Tycho, and Kepler took the existence of the planets for granted, and sought to learn how they move, and it was Newton who finally gave us dynamical laws for their motions. One is tempted to proclaim, existence precedes dynamics!

The reason this is misleading is obvious from consideration of how locations and motions were attributed to the planets in the first place. It was taken for granted by all those researchers that the planets were located in the regions from which they reflected light. That is, certain aspects of the way that these bodies interact with things were used to attribute to them spatial relations to other things, and then these spatial relations were used to investigate the ways in which their locations (or, rather, relative locations) changed with time. A dynamically inert object could not be said to be located anywhere, or to have distances from other dynamically inert things.

\section{Schematizing experimentation in quantum me- chanics}

Now let us take a look at how one might go about schematizing experiments and their results, within quantum mechanics, with an eye to what goes wrong.

First, a few words about how one sets up a quantum representation of a system, say, a hydrogen atom, to invoke the theory's first serious physical application.

One first identifies some dynamical variables of the system of interest, for example, position and momentum of the electron and of the proton that make up a hy- 
drogen atom. One then associates with these variables operators - Dirac called them " $q$-numbers" - on which are imposed the appropriate commutation relations. One then constructs a Hilbert space for the operators to act upon. A (pure) state of the system in which it has a given value of a dynamical variable is represented by a vector that is an eigenvector of the corresponding operator. This is readily extended to mixed states; a mixture of pure states that are represented by eigenvectors of an operator with a common eigenvalue is a state in which the system has the corresponding value of the dynamical variable. This interpretational rule, which is half of the eigenstateeigenvalue link, is the primary means by which a Hilbert space vector can be taken to indicate anything at all about a physical system. Thus, for example, if a hydrogen atom is in a state in which its total energy is $E$, that fact is indicated by taking the vector that represents its state to be an eigenvector, with eigenvalue $E$, of the operator associated with the total energy of the system. ${ }^{3}$

It is useful to distinguish between the two halves of the eigenstate-eigenvalue link. Let us call the conditional, "If the state of a system is an eigenstate of an operator corresponding to some dynamical quantity, the system possesses the corresponding eigenvalue" the positive eigenstate-eigenvalue link, and its converse, obtained by replacing "if" with "only if," the negative eigenstate-eigenvalue link. The positive link is indispensable for interpreting the quantum formalism as any sort of physical theory at all; the negative eigenstate-eigenvalue link leads to trouble.

Some experiments double as preparation procedures. These are the repeatable experiments, those that, if repeated on the same system, are sure to yield the same result. It is natural to suppose that, after such an experiment, the system indeed possesses the corresponding value of the variable in question. And, indeed, this was introduced as an explicit postulate, the collapse postulate, in von Neumann's Mathematische Grundlagen der Quantenmechanik (1932), whence it made its way into the second edition (1935) of Dirac's Principles of Quantum Mechanics (it does not appear in the first edition of 1931). In Dirac's formulation, "a measurement always causes the system to jump into an eigenstate of the dynamical variable that is being measured, the eigenvalue this eigenstate belongs to being equal to the result of the measurement" (Dirac, 1935, p. 36).

How are we to schematize the process of performing an experiment in quantum theory? We schematize the experimental apparatus as a quantum system with at least as many distinguishable states as there are distinguishable outcomes of the experiment. Among its dynamical degrees of freedom there is a "pointer observable" whose role it is to indicate the outcome. We assume an interaction between the system of interest and the apparatus that is such that, if the initial state of the system of interest is an eigenstate of the quantity to be "measured," then the apparatus will end up indicating it, which is taken to mean: the apparatus will end up in the appropriate eigenstate of the pointer observable. And therein lies the problem. Unless we assume a collapse postulate, the interaction between the system and apparatus, when the initial state of the system is not an eigenstate of the quantity to be "measured," will lead to an entangled state of system and apparatus that is not an eigenstate of the pointer observable. The eigenstate-eigenvalue link leaves it somewhat mysterious what we

\footnotetext{
${ }^{3}$ David Wallace (2013, p. 215) has claimed that "the E-E link has nothing much to do with quantum theory" and that "it seems to be purely an invention of philosophers" (Wallace, 2012, p. 4580). Gilton (2016) has decisively shown, with ample documentation of the relevant texts, that this is false.
} 
are to make of such a state. The prescription that, if the state of the apparatus is an eigenstate of the pointer observable, the value of the pointer observable is the corresponding eigenvalue, tells us nothing about what to say when the state is not an eigenstate. If we add the converse, "only if," then we conclude that the pointer is not pointing to any result, and that experiments don't have outcomes, which is hard to make sense of. We can, of course, deny that the pointer observable has a definite value only if the apparatus's quantum state is an eigenstate of the pointer observable; this leads us to posit structure beyond the quantum state.

The quantum state monist, if she is to hold on to the usual assumption that experiments have unique definite outcomes, must modify the dynamics so as to avoid states in which the pointer fails to point. This is where the collapse postulate comes in. A dynamical collapse theory, taken as a candidate for a solution to the measurement problem, is meant to be a substitute for the problematic versions of the postulate found in textbooks, a substitute that avoids the textbook postulate's invocation of "measurement" or "experiment" as a primitive concept, and avoids also the problematic assumption of two distinct sorts of processes, those that transpire during a measurement, and those that transpire at all other times. That is, a theory of this sort is meant to provide, to borrow from GRW's title (Ghirardi et al., 1986), unified dynamics for microscopic and macroscopic systems.

\section{Ontology for ideal collapse theories}

Let us entertain, for the duration of this section, the fiction that we could construct a dynamical collapse theory that succeeded in producing, and maintaining, eigenstates of some physical quantity. Which variables should these be, in order for the theory to be capable of yielding a world full of things to be met with in space? And, if we could choose an appropriate quantity, would this be sufficient, or would there still be something missing from the world?

Ghirardi et al. (1995) argue that a smeared mass density fills the bill. To avoid complications arising from relativity, suppose we have a nonrelativistic quantum field theory (that is, it permits particle creation and annihilation), containing particle types that may be indicated with an index $k$ (associated with each type is a characteristic mass, charge, spin, etc.). Let $\hat{\psi}_{k}^{\dagger}(\mathbf{x}, t), \hat{\psi}_{k}(\mathbf{x}, t)$ be creation and annihilation operators for particles of type $k$, satisfying the usual bosonic or fermionic commutation or anticommutation relations, and define particle number density operators by

$$
\hat{N}^{(k)}(\mathbf{x}, t)=\hat{\psi}_{k}^{\dagger}(\mathbf{x}, t) \hat{\psi}_{k}(\mathbf{x}, t) .
$$

We can use these to define smeared number operators. Given a non-negative, realvalued function $g$ with

$$
\int_{-\infty}^{\infty} d^{3} \mathbf{x} g(\mathbf{x})=1
$$

we define

$$
\hat{N}_{g}^{(k)}(\mathbf{r})=\int_{-\infty}^{\infty} d^{3} \mathbf{x} g(\mathbf{x}-\mathbf{r}) \hat{N}_{k}(x) .
$$

To get a feel for this, if $g(\mathbf{x})$ is taken to be constant within a sphere of radius $a$ centred on the origin, and zero outside it, then $\hat{N}_{g}^{(k)}(\mathbf{r})$ would be the operator corresponding 
to type- $k$ particle number in a sphere of radius $a$. That is, its expectation value, in any quantum state, would be the expectation value of the result of an experiment that counts the number of $k$-type particles in that sphere. An eigenstate of this operator would be one in which there is a definite number of $k$-type particles in the sphere.

Given a smearing function $g$, one can define smeared mass density operators by weighting particle numbers with their masses and summing over all particle types:

$$
\hat{M}_{g}(\mathbf{r})=\sum_{k} m_{k} \hat{N}_{g}^{(k)}(\mathbf{r}) .
$$

There are principled reasons for choosing variables of this sort as the ones that a collapse theory will tend to make definite; see Ghirardi et al. (1995) and Bassi and Ghirardi (2003) for discussion. The mass density should be smeared over distances small on a macroscopic scale, but large compare to atomic dimensions to avoid excessive narrowing of wave packets, to keep the unavoidable energy increase that accompanies collapse within acceptable limits.

For a toy version of a smearing of this sort, partition all of space into disjoint cubes with sides of a length $a$, a length that is small on the macroscopic scale but large compared to atomic dimensions - say, $10^{-5} \mathrm{~cm}$., which is small on the human scale of things but is nevertheless 10,000 times the Bohr radius, and, for each of these cubes, define a smeared mass density operator via a function that is uniform over that cube. Let us imagine a dynamical collapse theory whose stable states are eigenstates of mass density smeared uniformly over these cubes, and tolerates superpositions of distinct values of these smeared mass densities for time intervals that are minuscule on the human time scale. Could this theory describe a world of macroscopic objects anything like our own?

The answer, I think, is yes, but some argumentation is required to reach this. There is a temptation to think the answer is simple. The theory will include stable states in which there is a table-shaped region in this room of mass density higher than its surroundings, and might be tempted to say that we can simply declare that, according to the theory, that is what it is to be a table. But this is too quick, because we need assurance that this table-shaped region has the right sort of dynamical properties to make it a table. Does it reflect light? Of what frequencies? What happens if I place my mug-shaped region of high mass density on the upper boundary of the table-shaped region?

These questions have some urgency because the dynamical properties of a table - its stability, its capacities to reflect light and to support mugs, and the like - are chiefly a matter of its electromagnetic interactions with other things, and to specify a mass density and to say nothing more is to say nothing about electromagnetic properties. One could consider also smeared charge densities, but, since our cell size is large compared to atomic dimensions, for ordinary matter charge densities smeared over such regions will be zero or near-zero. Nonetheless, the electromagnetic properties of ordinary objects are crucial to their being what they are. A table-shaped region of high mass density composed entirely of electrically neutral matter would not hold its shape except momentarily, nor would it reflect light or support a mug, which would pass right through it without resistance.

An analysis that pays heed to the details of the quantum state yields the correct answer. A table is not merely a mass density located in a table-shaped region, but a structure composed of nucleons and electrons in bound states, forming atoms, which 
are bound together in a more-or-less rigid configuration. Our quantum state, which, by supposition, localizes a mass corresponding to definite integral multiples of particle masses in each of these cubes, will be one such that includes many-body correlations between the positions of the various constituents. Even if the location of a single nucleon is somewhat indefinite within the cube, it will be correlated with the positions of the other nuclei that make up the atomic nucleus of which it is a part, and the position of the nucleus of the atom will be correlated with its electrons. Thus, even though the smeared charge density might be zero within a cube, and even if there is not a definite matter of fact about the charge distribution within the cube, it will be a matter of fact that the charge is not uniformly zero within the cube, and that positive charges corresponding to atomic nuclei are surrounded by clouds of negative charge. This means that a photon that impinges on a table-region will interact primarily with outer shell electrons, and be absorbed or reflected. It also means that interactions between my mug-shaped region and the table-shaped region will be dominated by outer shell electrons, and that they will repel each other at short range.

Thus, the image we get from the theory, of regions of high mass density that behave much like we expect classical objects to behave, depends heavily on features that we regard as peculiarly quantum. In order for there to be stable atoms and molecules, let alone stable macroscopic objects, it is necessary for electron wave functions to be spread out over atomic and molecular distances. It also depends on quantum multiparticle correlations; though the nucleus of an atom might have its center-of-mass wave function spread out over a region of the size of the localization scale, there will be correlations on a finer scale between the positions of the individual nucleons, and between the positions of the nucleons and their accompanying electrons.

Upshot of this: A collapse theory that yielded eigenstates of mass density smeared over regions that are small on the macroscopic scale but large enough to permit internal goings-on at the atomic scale to proceed unmolested, would, indeed, solve the problem of yielding a description of a world containing things to be met with in space, but this is dependent on the physical reality of features of the quantum state that go well beyond the mass density it represents. The other features of the world represented by the quantum state are an integral part of the ontology.

\section{Ontology for near-collapse theories}

\subsection{The fuzzy link}

In the previous section, we entertained the fiction of a collapse theory that yielded eigenstates of the mass content of the elements of a partition of space into cubes that are small on the macroscopic scale, and argued that, in conjunction with other aspects of quantum theory, a theory like that can, indeed, yield an adequate description of a world containing things to be met with in space. On such a theory, there is a matter of fact about how much mass there is in each of the little cubes. The theory admits states in which there are regions of high mass density that have the requisite dynamical properties to count as bodies in the Newtonian sense.

Instead of this fictional theory, let us consider the actual Continuous Spontaneous Localization theory (CSL) (Pearle 1989, Ghirardi, Pearle, and Rimini 1990). This differs in two ways from our fictional theory. One is that, instead of a uniform smearing 
function over a precise region, Ghirardi, Pearle, and Rimini employ a smooth smearing function, a spherical Gaussian, characterized by a width $a$. The corresponding smeared number operators, which we will call simply $\hat{N}^{(k)}(\mathbf{r})$, can be thought of as an average number of particles in an imprecisely defined region of roughly size $a^{3}$. The other difference is that the theory does not produce eigenstates of the corresponding smeared mass-density operators, but, instead, tends to suppress superpositions of states in which there is a substantial spread in the value. That is, it will tend to suppress (but not completely eliminate) superpositions of states that correspond to differences in these smeared mass densities.

To get a feel for this, consider two macroscopic regions, $A$ and $B$, separated by a macroscopic distance. Let $\left|\psi_{A}\right\rangle$ be a state in which there is a large number $N$ of atoms mostly localized in $A$. That is, the many-particle wave-function for this state, $\psi_{1}\left(\mathbf{x}_{1}, \ldots, \mathbf{x}_{N}\right)$, has non-negligible amplitude only when all of $\mathbf{x}_{\mathbf{1}}, \ldots, \mathbf{x}_{N}$ are in $A$. Let $\left|\psi_{B}\right\rangle$ be a state in the same number of atoms are localized in $B$. The states $\left|\psi_{A}\right\rangle$ and $\left|\psi_{B}\right\rangle$ could be states that are close to eigenstates of the smeared mass density operators everywhere, but an equally weighted superposition of the two, or any superposition with non-negligible coefficients for both of these, would not be close to an eigenstate of $\hat{N}(\mathbf{r})$ for $\mathbf{r}$ within the respective regions in which the states $\left|\psi_{A}\right\rangle$ and $\left|\psi_{B}\right\rangle$ localize the particles. A state like that would tend to collapse into one in which most of the mass density is in either $A$ or $B$.

Most, but not all; the theory produces only approximations to eigenstates of the smeared mass density operators. The fact that collapse theories do not produce eigenstates of anything like our familiar physical quantities, but at best approximations to them, has been called the "tails problem." 4 This feature of collapse theories motivated, on the part of the developers of such theories, a modification of the eigenstateeigenvalue link.

if one wishes to attribute objective properties to individual systems one has to accept that such an attribution is legitimate even when the mean value of the projection operator on the eigenmanifold associated to the eigenvalue corresponding to the attributed property is not exactly equal to 1 , but is extremely close to it (Ghirardi et al. 1990, p. 1298; see also Ghirardi, Grassi, and Pearle 1991, p. 114, Ghirardi and Pearle 1991, p. 39, Ghirardi, Grassi, and Benatti 1995, p. 13).

This modification has been dubbed, by Clifton and Monton (1999), the fuzzy link. ${ }^{5}$

A state $|\psi\rangle$ is an eigenstate of an operator $\hat{A}$ if and only if the variance of $\hat{A}$ in that state,

$$
\mathcal{V}(\hat{A})=\left\langle\psi\left|\hat{A}^{2}\right| \psi\right\rangle-\langle\psi|\hat{A}| \psi\rangle^{2},
$$

is equal to zero. One way to estimate closeness to being an eigenstate of some variable is to use the criterion that its variance be small. Ghirardi, Grassi, and Benatti (1995) apply this criterion to mass densities. They say that the smeared mass density in the

\footnotetext{
${ }^{4}$ This was first flagged as a problem by Shimony (1991), and by Albert and Loewer (1991).

${ }^{5}$ Peter Lewis (2016, pp. 86-90) distinguishes between a fuzzy link, according to which there is some precise threshold $p$ such a system possesses the property $A=a$ if and only if $\left\langle\Psi\left|P_{a}\right| \Psi\right\rangle>1-p$, and a vague link according to which possession of a definite property is a matter of degree. It is hard to imagine what arguments there could be (or even what it might mean) for there to be a precise threshold. Albert and Loewer (1996) argue, correctly in my opinion, that there could be no such precise threshold, and that the modified link must be somewhat vague. This is what I mean by a fuzzy link.
} 
vicinity of a point $\mathbf{r}$ is objective if and only if the variance of $\hat{M}(\mathbf{r})$ is small compared to the square of its mean value:

$$
\frac{\mathcal{V}(\hat{M}(\mathbf{r}))}{\langle\psi| \hat{M}(\mathbf{r}))|\psi\rangle^{2}} \ll 1 .
$$

In some later works (Ghirardi and Grassi, 1996; Ghirardi, 1997a) the mass density is said to be "accessible" if this criterion is satisfied.

How close is close enough? Obviously, to pick a precise fixed threshold would be every bit as arbitrary as picking a precise upper limit to Jupiter's atmosphere. The dynamics of the theory should be our guide, as well as our capacities to discriminate between quantum states. If the spread of some physical quantity is too small to be discernible, then the state's behaviour will not be appreciably different from that of an eigenstate, and we have license to disregard the difference. Ghirardi et al. (1995) justify the criterion by arguing that, when the criterion (6) is satisfied, things behave as if there is a classical mass in the smearing region equal to the expectation value of the smeared mass density.

This proposal is an application of the fuzzy link to the smeared mass densities. That is, it is a proposal about when, in the context of a dynamical collapse theory, we should regard the state as one in which we should say that there is a physical quantity that is sufficiently well-defined as to have, in effect, an objective value. It remains an interpretation on which "the statevector is everything" (Ghirardi and Grassi 1996, p. 368; see also Ghirardi 1997a, p. 364, Ghirardi 1997b, p. 233).

In a world like this, crucial physical quantities, such as the amount of mass in a given region of space, fail to have precise values, though they may come very close to having a precise value, in the sense of satisfying the accessibility criterion. Some readers will balk at this. A proposal of this sort, it might be said, offends intuition, or common sense. However, as we have said already in the introduction, we have no reason to take common sense or intuitions to be reliable guides as to how the world is. We should ask: can a case be made that, as a matter of conceptual necessity, the world cannot be like that?

Any attempt at such an argument is doomed to fail. It seems to me that anyone who accepts the following propositions, which strike me as incontrovertible, should accept that the world could, indeed, be like that.

- To be a physical body is nothing more and nothing less than to have a certain place in a network of dynamical and causal relations of the sort discussed in section 3 .

- An ideal collapse theory, if there could be one, on which sufficiently large regions of space contained perfectly definite quantities of mass, would, provided that the dynamics of the theory underwrote the right sort of dynamical behaviour of these masses, would be capable of delivering a sensible description of a world much like ours.

- Given the dynamics of a theory like CSL, a small difference in a state makes only a small difference to dynamical behaviour.

This is an application of a more general Principle of Continuity for metaphysics. If certain states of a physical theory are capable of bearing some metaphysical burden, 
then a slightly different state is capable of bearing the same burden. If this continuity principle were not accepted, it seems to me, then we would not be capable in engaging in metaphysics at all, as any physical theory we have ever formulated, and any physical theory that we are likely to formulate in the foreseeable future, is at best some sort of approximation to a deeper, more fundamental theory. And, even if we were handed an exact theory on tablets of stone, we would still only know the physical states of things within a certain degree of approximation.

This perspective on ontology - that it is really how things interact with other things that counts - suggests a natural choice for the variables that the collapse theory will tend to make definite. As interactions can be represented by terms in a complex system's Hamiltonian, a natural choice would be energy density, or, in a relativistic context, the stress-energy tensor. In a non-relativistic approximation, most of a massive object's energy is in its rest mass, and so the energy density would approximate a mass density, and arguments that a mass density makes for an adequate ontology would carry over.

\subsection{Adding primitive ontology}

There is in the literature another option, which has become more popular in recent years, for interpreting collapse theories.

For any state $|\Psi\rangle$, whether or not it is at all close to an eigenstate of any of the operators $\hat{M}(\mathbf{r})$, one can define the expectation value.

$$
m(\mathbf{r})=\langle\Psi|\hat{M}(\mathbf{r})| \Psi\rangle .
$$

If one is dissatisfied with the fuzzy link, which involves vagueness at the level of basic ontology, an alternative is to postulate, as additional ontology over and above the quantum state, a mass density that always exists, and always has the value, at any time and place, given by (7). This can be thought of as introducing the mass density as primitive ontology. ${ }^{6}$ This option has the advantage of being more in accord with classical intuitions. The mass density, always definite, is like a classical mass density, with the chief difference that it is subject to unclassical dynamical laws. There's no question, intuition tells us, of what we're talking about, on a theory with this primitive ontology.

This, it seems to me, is an illusion. The reason is, essentially, precisely the reason that led Ghirardi and co-authors to distinguish between mass densities that are objective, or accessible, and those that are not.

The relation between quantum states and mass density functions is obviously manyone. A mass density function is uniquely determined by a quantum state, but the same mass-density function can correspond to many different quantum states. This is illustrated by an example discussed by Ghirardi et al. (1995). Consider two spherical regions, $A$ and $B$, and consider two quantum states. The first state to be considered, $\left|\Psi^{\oplus}\right\rangle$, is an equally weighted superposition of two states, $\left|\Psi_{N}^{A}\right\rangle$ and $\left|\Psi_{N}^{B}\right\rangle$. In $\left|\Psi_{N}^{A}\right\rangle$, a large, even number of particles, of total mass $M$, are all located (within the tolerances

${ }^{6}$ As far as I can tell, this proposal first appears, explicitly, in Goldstein (1998). It is discussed extensively in Allori et al. (2008). It should be mentioned that Ghirardi has adopted this view of the status of the mass density, and now regards it as "an additional element which need to be posited in order to have a complete and consistent description of the world" (Ghirardi, 2007, p. 2907). 
permitted by the theory) in the region $A$, and evenly distributed within it. $\operatorname{In}\left|\Psi_{N}^{B}\right\rangle$, the same holds of $B$. The state $\left|\Psi^{\oplus}\right\rangle$ is,

$$
\left|\Psi^{\oplus}\right\rangle=\frac{1}{\sqrt{2}}\left(\left|\Psi_{N}^{A}\right\rangle+\left|\Psi_{n}^{B}\right\rangle\right)
$$

The second state to be considered, $\left|\Psi^{\otimes}\right\rangle$ is one in which each of the regions $A, B$ contains half of the particles.

$$
\left|\Psi^{\otimes}\right\rangle=\left|\Psi_{N / 2}^{A}\right\rangle \otimes\left|\Psi_{N / 2}^{B}\right\rangle .
$$

The two states yield the same mass-density function, taking on a value corresponding to $\frac{1}{2} M$ in $A$ and $\frac{1}{2} M$ in $B$. But there is a difference between the physical conditions of the regions in those two states. This can be seen by the behaviour of a test particle projected along a line that passes directly between the two regions, and which is attracted gravitationally to the masses within those regions. In state $\left|\Psi^{\otimes}\right\rangle$, the particle, being attracted equally by both masses, passes undeflected. In state $\left|\Psi^{\oplus}\right\rangle$, by contrast, even though the mass density takes on the same values that it does in state $\left|\Psi^{\otimes}\right\rangle$, the reaction of the test particle is very different. If initially well-localized, its state will become entangled with that of the other masses, so that the state of the whole system ends up being a state that is a superposition of two terms, in one of which the test particle is deflected towards $A$, and in the other, deflected towards $B$.

In state $\left|\Psi^{\oplus}\right\rangle$, do each of the two regions contain a mass $\frac{1}{2} M$ ? It is not enough to simply declare this to be true; not even God could create masses in those regions in the absence of physical laws that ensure that they act like masses. And, in a state like $\left|\Psi^{\oplus}\right\rangle$, other things do not act as if there are those half-masses in those regions. Something that you might be inclined to call a "mass," if it doesn't act like a mass, is not a mass. There is no sense in which, in a state like $\left|\Psi^{\otimes}\right\rangle$, regions $A$ and $B$ each contain mass $\frac{1}{2} M$.

Only when the function defined by (7) acts like a mass density are we justified in calling it one. And this is only when it satisfies the criterion of "accessibility."

But this means that nothing is added by taking there to be additional ontology, beyond the quantum state. The mass density $m$ acts like a mass density only when the accessibility criterion is satisfied, and, when it does, the quantum state dynamics alone ensure that everything behaves as if a mass density were present. I am for this reason in agreement with Ghirardi and Grassi, when they write, ${ }^{7}$

For the reader who has followed the above analysis, it should be clear that, within CSL, physical systems are fully described by the state vector which evolves according to equations embodying genuine elements of chance. However, even though the state vector is everything ..., since the dynamics itself tends to make objective the mass density, it turns out to be appropriate to relate the kinematical elements in Bell's sense to the mass density distribution.

... In a sense we could state that the exposed beables of the theory are the values of the mass density function and that, at the appropriate level, the dynamics allows one to take them seriously. All other properties which

\footnotetext{
${ }^{7}$ Parallel passages, differing only in minor matters of wording, can be found in Ghirardi (1997a, pp. 363-364) and Ghirardi (1997b, p. 233).
} 
emerge as a consequence of this process derive from this basic dynamical feature concerning the mass density function (Ghirardi and Grassi, 1996, p. 368)

Though the mass density function is singled out by the dynamics as the one that is almost always "accessible," it does not exhaust what there is in the world. Other quantities, such as charge densities, could get carried along with the mass density, and, when they satisfy the criterion of accessibility, they are no less real than the mass density. ${ }^{8}$ A region of space that acts as if it contains a certain charge does contain that charge; no sense can be made of a claim that only the masses are real and that other physical quantities have an inferior ontological status. Their reality may be emergent as a consequence of the dynamics, but emergent is not the same as unreal.

To some readers, it will seem as if I am smuggling in unwarranted presuppositions. The thought would go something like this. In the absence of additional ontology, a quantum state is just an abstract object, a vector in a Hilbert space, or else something like a field on a high-dimensional space with no intrinsic relation to things in threedimensional space. It is only when additional ontology is introduced, ontology on three-dimensional space, and the quantum state placed in the role of guiding that ontology, that it has some relation to our three-dimensional space.

This line of thinking - which, admittedly, has become prevalent in the literatureseems to me to misconstrue the nature of quantum theories. We are not presented with an abstract Hilbert space, with no built-in relation to anything physical, or, worse with a mysterious function on a high-dimensional space. To construct a quantum theory, one first identifies a target system, with certain dynamical degrees of freedom. One then follows the quantization heuristic to construct a quantum theory of that system, replacing the canonical dynamical variables with operators obeying appropriate commutation relations. Insofar as the dynamical degrees of freedom are associated with certain regions of space, so are the operators that we associate with them, and the eigenvalues of those operators represent possible stable values of those quantities, and eigenstates of those operators represent states in which those values obtain in the appropriate regions of space. All we are doing is extending this representation, via continuity, to states that are not eigenstates.

If a quantum state were a vector in a Hilbert space that had no built-in association with dynamical quantities, there would be a serious problem with taking it to represent anything physical, as the intrinsic structure of such a space would not distinguish one vector from another. If a quantum state were a field on configuration space, with no built-in relation to dynamical quantities such as energy or charge density or the like, or, worse, if it were a field on a high-dimensional space with no built-in relation to our familiar three-dimensional space, then there would, indeed, be a serious problem with taking it to describe a world anything like ours. If that is what quantum states were, then we would, indeed, need to posit additional ontology above and beyond the wave function. But taking a quantum state to be something like that involves, it seems to me, an almost willful forgetting of what quantum theories are.

\footnotetext{
${ }^{8}$ Shan Gao $(2017, \S 6.2 .1)$ argues, along similar lines, for the reality of charge densities when a charged system is well localized and hence the state is an eigenstate (or close to it) of charge density.
} 


\subsection{A comment on "flash ontology"}

Goldstein (1998) and Allori et al. (2008) adapt a suggestion of John Bell to formulate an alternate primitive ontology for the GRW theory, which they call the "flash ontology." Bell describes his proposal as follows.

There is nothing in this theory but the wavefunction. It is in the wavefunction that we must find an image of the physical world, and in particular of the arrangements of things in ordinary three-dimensional space. But the wavefunction as a whole lives in a much bigger space, of $3 N$-dimensions. It has neither amplitude nor phase nor anything else until a multitude of points in ordinary three-space are specified. However, the GRW jumps (which are part of the wavefunction, not something else) are well localized in ordinary space. Indeed each is centered on a particular spacetime point $(\mathbf{x}, t)$. So we can propose these events as the basis of the 'local beables' of the theory. These are the mathematical counterparts in the theory to real events at definite places and times in the real world ... A piece of matter then is a galaxy of such events. As a schematic psychophysical parallelism we can suppose that our personal experience is more or less directly of events in particular pieces of matter, our brains, which events are in turn correlated with events in our bodies as a whole, and they in turn with events in the outer world (Bell 1987, in Bell 2004, pp. 204-205).

Unlike Bell, for whom the jumps, or hitting events, are "part of the wavefunction, not something else" (it would be better to say: are events in the evolution of the wavefunction), Allori et al. propose that these events be taken as self-subsistent primitive ontology, distinct from the wave function, whose role is to furnish a probability distribution over possible sequences of flashes. We then have two distinct physical theories, with the same stochastic equation of motion for the wave function: GRWm, which is the GRW theory with mass-density ontology, and GRWf, GRW theory with the flash ontology.

As Allori et al. note, a table or chair will be the locus of a great many flashes per second - they estimate an order of $10^{8}$ flashes per cubic centimeter for solid matter. On a smaller scale, the flashes would be rarer. In particular, on this theory, in a region of space that we are inclined to take to be the locus of a living cell, the interval between flashes will be of the order of $10^{3}$ seconds, something like one flash per hour. About this, in a footnote that responds to a comment by a referee, they write, "there is presumably no cell in GRWf, though the structure of the wave function (on configuration spaceeven though there are no configurations) might suggest otherwise" (p. 362, fn. 5).

Of course, this does not mean that, if we look through a microscope at that region in which there is no cell, we will see nothing. On the contrary, the photons or electrons, as the case may be, employed by the microscope will become entangled with the wave function of the non-existent cell, and the amplification process will eventually record the image in some medium involving a macroscopic number of atomic nuclei, enough so that the encoded image of a cell will be discernible in the pattern of flashes in the recording medium. We will have formed an image of something that does not exist.

Moreover, unlike the case of properties of quantum systems to which the quantum state assigns nothing like a determinate value prior to interaction with experimental apparatus - cases in which we should, indeed, say that the recorded result does not 
represent a fact that obtained prior to the interaction - it is possible to prepare the living cell in such a way that an observation will predictably and reliably produce an image of a cell in a given location.

We should ask: what are we saying, here? Can we coherently acknowledge that is true of a given region of space that it reacts, predictably and reliably, in these ways to microscopes and other instruments, and, indeed, to anything else that might interact with it, exactly as if a cell is present, and at the same time deny that there is a cell present? The supposedly nonexistent cell would (as Newton put it) no less constitute a part of the structure of things than the bodies realized in the primitive ontology. We are being asked to draw an ontological distinction between dynamical potentialities associated with regions in which there is primitive ontology, and those located in empty space, with only the former counting as real objects. It is as if an Aristotelian, rejecting the Newtonian conception of body, declared that the only genuine bodies were those in which the dynamical properties flagged by Newton were realized in some materia prima, and, further, declared that some of the apparent objects we interact with are of this sort, and others, mere simulacra. All this is, literally and without hyperbole, nonsensical.

\section{Distributional ontology}

Consideration of situations such as the one discussed in $\S 6.2$, above, makes it clear that specifying a mean mass distribution (even if this were reified into primitive ontology) does not exhaust the physical facts about mass distributions. Things are very different, depending on whether the variance $\mathcal{V}(\hat{M}(\mathbf{r}))$ is appreciable or not. We must, therefore, take it that there is a matter of fact about what this variance is.

This suggests the following revision, advanced by Philip Pearle (2009), to the way we attribute values to dynamical quantities. In classical physics, dynamical quantities always possess precise values. In quantum theory, there is always some imprecision, but, if the spread is small enough - that is, if the state is close enough to an eigenstate - we may treat it as one in which the quantity has a precise value. But the full reality is that associated with each dynamical variable is a distribution of values. This distribution, though formally like a probability distribution, is to be thought of, not as a probability distribution over a precise but unknown possessed value, but as reflecting a physical, ontological, lack of determinacy about what the value is.

On this proposal every dynamical variable possesses some distribution. A collapse theory will tend to narrow the spread of the distributions of some of these quantities, and, when the distribution is sufficiently narrow, everything will be almost exactly as if the quantity has a precise value, and it can be treated as if it were precise. If we are seeking objects that behave like our familiar macroscopic objects, it is to those variables that we should direct our attention. But the spread-out distributions of the other variables are no less part of physical reality.

An analogy might help. For many purposes, in dealing with solar system dynamics, the planets can be treated as if they have precise, point locations. They don't, of course. Suppose, then, we wanted to develop a more nuanced concept of location of Jupiter. One refinement is to note that Jupiter is extended, and we could choose a certain region, an oblate spheroid, as the region in which Jupiter is located. But Jupiter's atmosphere does not have a precise upper boundary. The location of Jupiter is neither 
a determinate point in space nor a determinate region of space.

On this proposal, all dynamical physical quantities are like that. Take a spatial region $R$, and consider the quantity of mass in that region, $M(R)$. Classically, this would have a point value. On the distributional ontology, it does not have a precise value, but might have, instead, a distribution ${ }^{9}$ whose density function looks something like that depicted in Figure 1. A state in which the distribution for $M(R)$ was like that would be nearly an eigenstate of the property corresponding to $M(R)$ lying within the interval $[0.5,1.5]$. It would be even more nearly an eigenstate of the property corresponding to $M(R)$ lying within the interval $[0,2]$. For any interval $\Delta$ of the real line, we can consider the projection onto the subspace that, by the positive eigenstateeigenvalue link, corresponds to $M(R)$ being in $\Delta$. Any state vector can be written as a sum of a component within that subspace and a component orthogonal to that subspace If the ratio of the norm of the component within that subspace to the norm of the state vector is close enough to unity, close enough for the difference to be negligible, then we are justified in asserting that the value of $M(R)$ lies in that interval.

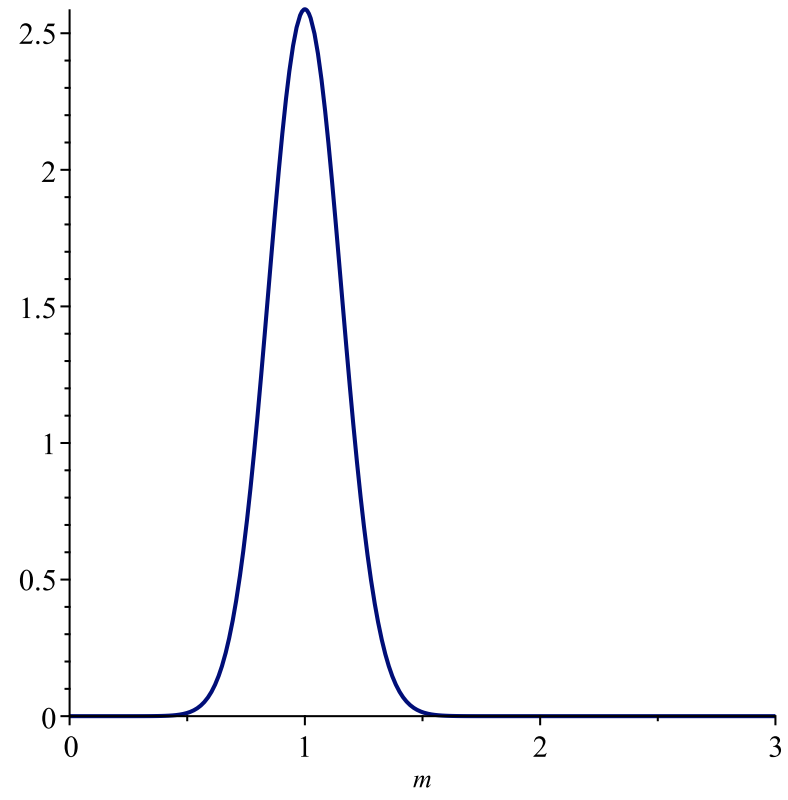

Figure 1: A hypothetical density function for a mass-value distribution

Such assertions will be somewhat elliptical, as they carry an implicit degree of tolerance, which can be made explicit. That is, assertions that the value of a physical quantity lies within a given interval are really what Philip Pearle (2009) calls qualified possessed value attributions.

Consider a state $|\psi\rangle$ for which

$$
\frac{\| \hat{P}(M(R) \in \Delta)|\psi\rangle \|^{2}}{\||\psi\rangle \|^{2}} \geq 1-\varepsilon,
$$

\footnotetext{
${ }^{9}$ Don't confuse this distribution, which is a distribution of values for the mass within a fixed region of space, with a mass distribution that attributes various mass-contents to various regions of space.
} 
where $\hat{P}(M(R) \in \Delta)$ is the projection onto the subspace for which $M(R)$ is in the interval $\Delta$, and $\varepsilon$ is some small number (say $10^{-40}$, as suggested by Pearle (1997)). Applying the usual probability rules to outcomes of experiments, the probability in such a state that a measurement of mass in $R$ will yield a value within $\Delta$ is at least $1-10^{-40}$, which means that " 100 billion people making measurements every picosecond for 10 billion years can expect only one erroneous prediction" (Pearle, 1997, p. 151). This seems enough to warrant the claim that we can reliably predict the result of such an experiment, and that there is a fact of the matter about the region $R$, that it will respond in the affirmative to an experiment to determine whether its mass content is in $\Delta$.

However, in our attribution of properties to physical systems, we should not take "experiment" as a primitive term. ${ }^{10}$ Worse, this way of speaking might be taken to presume that experimental outcomes have unqualified definite values, whereas, on this account, every physical quantity, including the pointer variables of our experimental apparatus, is distribution-valued. We should be a bit careful about what it means, on this account, to say that we can reliably predict the result of an experiment.

A good experimental apparatus is one such that the dynamics of the theory will lead to near-sharp pointer values. Moreover, the sharpness of such pointer values is limited only by the resources we have available to us in building the apparatus. So, when we attribute a qualified possessed value to the proposition $M(R) \in \Delta$ with tolerance $\varepsilon$ in a state $|\psi\rangle$, the operational import of this is the following.

For any $\delta>0$, no matter how small, it is possible for there to be another system, with an indicator variable $\Pi$, whose spectrum includes indicator regions $\Pi^{+}$and $\Pi^{-}$, such that:

1. If applied to an eigenstate of $M(R) \in \Delta$, the indicator variable will end up in $\Pi^{+}$within tolerance $\delta$.

2. If applied to an eigenstate of $M(R) \notin \Delta$, the indicator variable will end up in $\Pi^{-}$within tolerance $\delta$.

3. If applied to the state $|\psi\rangle$, with probability at least $1-\varepsilon$, the indicator variable will end up in $\Pi^{+}$within tolerance $\delta$.

That is, we attribute properties to systems on the basis of their potential responses to other systems, and the tolerances invoked have to do with probabilities of various responses. Those responses, in turn, will be responses of distribution-valued variables, and will be themselves the subjects of qualified value attributions.

Is there a threat of circularity here? There might be, if we had no handle on the meaning of any of these value attributions. But they are anchored by the positive eigenstate-eigenvalue link. As the tolerance $\varepsilon$ shrinks, a state in which a certain dynamical quantity has a given value within a tolerance $\varepsilon$ approaches an eigenstate with that eigenvalue, and, as a consequence of that, its behaviour approaches that of the eigenstate. Recall that our quantum theory was set up in the first place with an identification of certain dynamical variables of interest, and an association of operators with

\footnotetext{
${ }^{10}$ This is why I choose to say that qualified value attributions are qualified by specifying a tolerance, whereas Pearle speaks of them as qualified by the probability that the statement is false. There is nothing wrong with this way of speaking, if it is understood in the way that is outlined below, but it runs the risk of suggesting that we are taking experiment as an unanalyzed primitive, and not something to be schematized within the theory.
} 
those variables. We know what it means to attribute to a system a definite value of some dynamical quantity. To attribute an almost-definite value means: it behaves in almost the same way as it would if it had the definite value.

\section{Conclusion}

A distributional ontology of the sort outlined above is, I claim, the natural way to think about a world described by a dynamical collapse theory, if one takes the theory seriously, on its own terms. Attributions of values to physical quantities will always be qualified value attributions. The rationale for accepting qualified possessed value attributions is that, as $\varepsilon$ decreases without limit, any dynamical differences between a state in which a quantity is possessed within tolerance $\varepsilon$ and a state in which it is possessed with zero tolerance - that is a state that, in accordance with the eigenstateeigenvalue link, the quantity is possessed without qualification - diminish without limit. On the Newton-inspired conception of body, what it is for there to be bodies with spatial locations (or even relative spatial locations) has to do with dynamical properties associated with those locations, and there is nothing in that conception of body that requires those properties to be point-valued.

This may run counter to some readers' intuitions. Those intuitions might long for an ontology on which everything is definite. They may long for an ontology in which dynamical properties are predicated of some substrate that is conceptually capable of being stripped of its dynamical properties. But neither the world nor physical theory are in any obligation to conform to such intuitions. A world like ours could be the way that a dynamical collapse theory says it is, and we should seriously entertain the possibility that it is that way. 


\section{References}

Albert, D. and B. Loewer (1991). Wanted dead or alive: Two attempts to solve Schrödinger's paradox. In A. Fine, M. Forbes, and L. Wessels (Eds.), PSA 1990: Proceedings of the 1990 Biennial Meeting of the Philosophy of Science Association, Volume One: Contributed Papers, pp. 2787-285. East Lansing, MI: Philosophy of Science Association.

Albert, D. and B. Loewer (1996). Tails of Schrödinger's cat. In R. Clifton (Ed.), Perspectives on Quantum Reality. Dordrecht: Kluwer Academic Publishers.

Allori, V. (2013). Primitive ontology and the structure of fundamental physical theories. See Ney and Albert (2013), pp. 58-75.

Allori, V., S. Goldstein, R. Tumulka, and N. Zanghì (2008). On the common structure of Bohmian mechanics and the Ghirardi-Rimini-Weber theory. The British Journal for the Philosophy of Science 59, 353-389.

Allori, V., S. Goldstein, R. Tumulka, and N. Zanghì (2014). Predictions and primitive ontology in quantum foundations: A study of examples. The British Journal for the Philosophy of Science 65, 323-352.

Bassi, A. and G. Ghirardi (2003). Dynamical reduction models. Physics Reports 379, $257-426$.

Bell, J. S. (1987). Are there quantum jumps? In C. Kilmister (Ed.), Schrödinger: Centenary celebration of a polymath, pp. 41-52. Cambridge: Cambridge University Press. Reprinted in Bell (2004, 201-212).

Bell, J. S. (2004). Speakable and Unspeakable in Quantum Mechanics (2nd ed.). Cambridge: Cambridge University Press.

Clifton, R. and B. Monton (1999). Losing your marbles in wavefunction collapse theories. The British Journal for the Philosophy of Science 50, 697-717.

Dirac, P. A. M. (1935). Principles of Quantum Mechanics (2nd ed.). Oxford: Oxford University Press.

Esfeld, M. (2014). Quantum Humeanism, or: Physicalism without properties. The Philosophical Quarterly 64, 453-470.

Esfeld, M., D. Lazarovici, V. Lam, and M. Hubert (2017). The physics and metaphysics of primitive stuff. The British Journal for the Philsophy of Science 68, 133-161.

Gao, S. (2017). The Meaning of the Wave Function. Cambridge: Cambridge Univerity Press.

Ghirardi, G. (1997a). Quantum dynamical reduction and reality: Replacing probability densities with densities in real space. Erkenntnis 45, 349-365. 
Ghirardi, G. (1997b). Macroscopic reality and the dynamical reduction program. In M. L. D. Chiara, K. Doets, D. Mundici, and J. V. Benthem (Eds.), Structures and Norms in Science: Volume Two of the Tenth International Congress of Logic, Methodology, and Philosophy of Science, pp. 221-240. Dordrecht: Kluwer Academic Publishers.

Ghirardi, G. (2007). Some reflections inspired by my research activity in quantum mechanics. Journal of Physics A: Mathematical and Theoretical 40, 2891-2917.

Ghirardi, G. and R. Grassi (1996). Bohm's theory versus dynamical reduction. In J. T. Cushing, A. Fine, and S. Goldstein (Eds.), Bohmian Mechanics and Quantum Theory: An Appraisal, pp. 353-377. Berlin: Springer.

Ghirardi, G., R. Grassi, and P. Pearle (1990). Relativistic dynamical reduction models: General framework and examples. Foundations of Physics 20, 1271-1316.

Ghirardi, G., R. Grassi, and P. Pearle (1991). Relativistic dynamical reduction models and nonlocality. In P. Lahti and P. Mittelstaedt (Eds.), Symposium on the Foundations of Modern Physics 1990, pp. 109-123. Singapore: World Scientific.

Ghirardi, G. and P. Pearle (1991). Elements of physical reality, nonlocality and stochasticity in relativistic dynamical reduction models. In A. Fine, M. Forbes, and L. Wessels (Eds.), PSA 1990: Proceedings of the 1990 Biennial Meeting of the Philosophy of Science Association, Volume Two: Symposia and Invited Papers, pp. 35-47. East Lansing, MI: Philosophy of Science Association.

Ghirardi, G., A. Rimini, and T. Weber (1986). Unified dynamics for microscopic and macroscopic systems. Physical review D 34, 471-491.

Ghirardi, G. C., R. Grassi, and F. Benatti (1995). Describing the macroscopic world: Closing the circle within the dynamical reduction program. Foundations of Physics 25, 5-38.

Ghirardi, G. C., P. Pearle, and A. Rimini (1990). Markov processes in Hilbert space and continuous spontaneous localization of systems of identical particles. Physical Review A 42, 78-89.

Gilton, M. J. (2016). Whence the eigenstate-eigenvalue link? Studies in History and Philosophy of Modern Physics 55, 92-100.

Goldstein, S. (1998). Quantum theory without observers-Part two. Physics Today 51, $38-42$.

Lewis, P. J. (2016). Quantum Ontology: A Guide to the Metaphysics of Quantum Mechanics. Oxford University Press.

Lewis, P. J. (2017). On the status of primitive ontology. This volume.

Maudlin, T. (2007). Completeness, supervenience and ontology. Journal of Physics A: Mathematical and Theoretical 40, 3151-3171. 
Newton, I. (1962). Unpublished Scientific Papers of Isaac Newton: a Selection from the Portsmouth Collection in the University Library, Cambridge. Cambridge: Cambridge University Press. Chosen, edited, and trasnlated by A. Rupert Hall and Marie Boas Hall.

Newton, I. (1999). The Principia: Mathematical Principles of Natural Philosophy. Berkeley: The University of Cailfornia Press. Translated by I. Bernard Cohen and Anne Whitman.

Ney, A. and D. Z. Albert (Eds.) (2013). The Wave Function: Essays on the Metaphysics of Quantum Mechanics. Oxford University Press.

Pearle, P. (1989). Combining stochastic dynamical state-vector reduction with spontaneous localization. Physical Review A 39, 2277-2289.

Pearle, P. (1997). Tales and tails and stuff and nonsense. In R. S. Cohen, M. Horne, and J. Stachel (Eds.), Experimental Metaphysics: Quantum Mechanical Studies for Abner Shimony, Volume One, pp. 143-156. Dordrecht: Kluwer Academic Publishers.

Pearle, P. (2009). How stands collapse II. In W. C. Myrvold and J. Christian (Eds.), Quantum Reality, Relativisitic Causality, and Closing the Epistemic Circle: Essays in Honour of Abner Shimony, pp. 257-292. Springer.

Shimony, A. (1991). Desiderata for a modified quantum mechanics. In A. Fine, M. Forbes, and L. Wessels (Eds.), PSA 1990: Proceedings of the 1990 Biennial Meeting of the Philosophy of Science Association, Volume Two: Symposia and Invited Papers, pp. 49-59. East Lansing, MI: Philosophy of Science Association. Reprinted in Shimony (1993, pp. 55-67).

Shimony, A. (1993). Search for a Naturalistic Wordlview, Volume II: Natural Science and Metaphysics. Cambridge University Press.

Stein, H. (1994). Some reflections on the structure of our knowledge in physics. In D. Prawitz, B. Skyrms, and D. Westertåhl (Eds.), Logic, Methodology, and Philosophy of Science IX, pp. 633-655. Amsterdam: Elsevier Science B.V.

Tumulka, R. (2017). Paradoxes and primitive ontology in collapse theories of quantum mechanics. This volume.

von Neumann, J. (1932). Mathematische Grundlagen der Quantenmechanik. Berlin: Springer Verlag.

Wallace, D. (2012). Decoherence and its role in the modern measurement problem. Philosophical Transactions of the Royal Society A 370, 4576-4593.

Wallace, D. (2013). A prolegomenon to the ontology of the Everett interpretation. See Ney and Albert (2013), pp. 203-222. 